\title{
Establishing order from chaos
}

DOI:

10.1038/nrc1899

URLs
Multiple myeloma (MM) remains largely incurable, with only $10 \%$ of patients surviving 10 years after diagnosis. Part of the problem is that MM is a highly heterogeneous disease, characterized by many changes in chromosome structure and number. Ron DePinho and colleagues sought to distinguish the cancer-associated changes in MM more fully by using an integrated oncogenomic approach.

$\mathrm{MM}$, which arises because of a clonal proliferation of plasma (antibody-secreting) cells, can be split into two categories: non-hyperdiploid and hyperdiploid, the latter of which tends to have a better prognosis. DePinho and colleagues initially used high-resolution array comparative genomic hybridization (aCGH) to identify copy-number aberrations in plasma cells from 67 newly diagnosed patients with MM. The authors developed an algorithm based on unsupervised methodologies and were able to group the aCGH results into a maximum of 4 distinct molecular subclasses $(\mathrm{k} 1-\mathrm{k} 4)$. This provided molecular evidence that $\mathrm{MM}$ is a heterogeneous disease and that the MM hyperdiploid class can be further subdivided into two molecular subclasses (k1-k2).

The authors then asked whether there was any difference in the survival of patients grouped as $\mathrm{k} 1$ or $\mathrm{k} 2-\mathrm{k} 1$ patients showed longer event-free survival and, to a lesser extent, overall survival. So, what are the chromosomal changes that determine this outcome? Comparison of the $\mathrm{k} 1$ and $\mathrm{k} 2$ genomic patterns identified several prominent changes indicating that a gain of (chromosome 11 ch11) is associated with a favourable outcome, but a gain of ch1q and/or loss of ch13 is associated with a poor clinical outcome.

Further evidence of the biological significance of the $\mathrm{k} 1$ and $\mathrm{k} 2$ subclasses was provided by gene-set enrichment analysis of the relevant transcriptomes. Although TP53, KRAS, FRAP and components of the proteasome pathway were altered in both k1 and k2 subgroups, deregulation of pathways such as sonic hedgehog, and deregulation of $R A C 1$ were only seen in the $\mathrm{k} 2$ samples. These findings need further validation.

However, not all of the important changes involved gross chromosomal alterations, so the authors also used the aCGH data to look for discrete minimal common regions (MCRs) that contained recurrent, highly focal copy-number alterations. Eighty-seven of the most disease relevant MCRs comprised 47 DNA amplifications and 40 DNA deletions. Fourteen of these MCRs were associated with poor survival.

As copy-number alterations influence gene-expression levels, the authors used integrated RNAexpression analyses to look for oncogenic expression patterns for every gene altered in an MCR. Of the 2,151 genes analysed, $30 \%$ were significantly overexpressed, narrowing down the search for oncogenic candidates in MM. These included genes with a known function in MM such as MYC, $A B L 1$ and MCL1, and other genes with no previous link to MM, including anaphase-promoting complex subunit 2 and F-Box protein 3 , and many genes involved in ribosome biogenesis and protein synthesis.

Interestingly, the use of this 'omics' approach has identified a large number of amplifications and deletions in which the cancer-relevant gene is not yet known. Therefore the authors suggest that there might be many cancer genes that are yet to be discovered that could provide new therapeutic targets as well as useful prognostic markers, for MM.

\section{Nicola McCarthy}

ORIGINAL RESEARCH PAPER Carrasco, D. R. et al. High-resolution genomic profiles defines distinct clinico-pathogenetic subgroups of multiple myeloma patients. Cancer Cell 9 , 313-325 (2005).

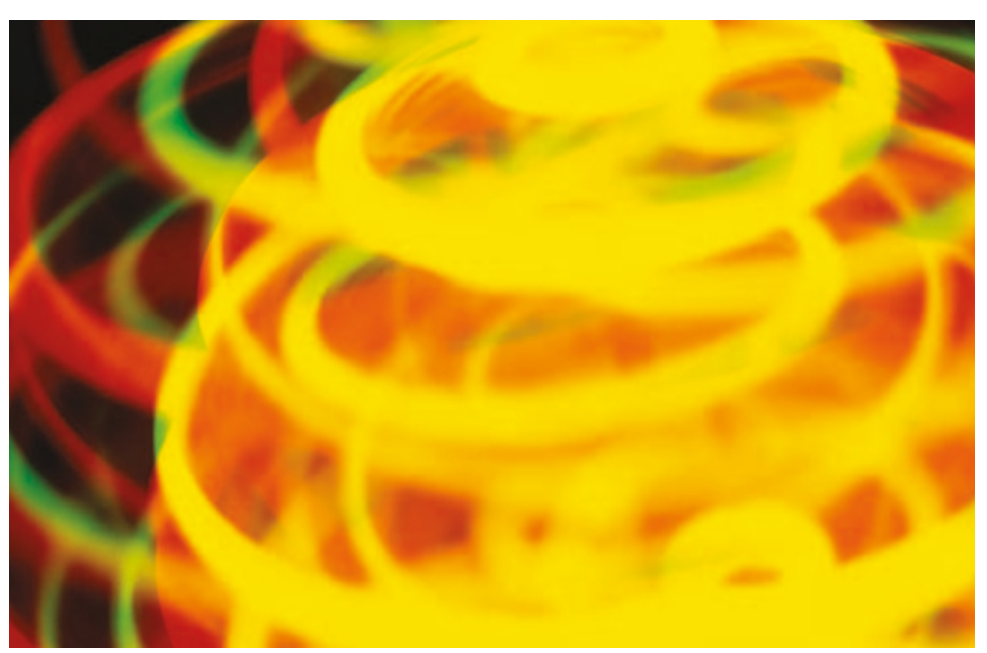

